\title{
Evaluation of in vitro virulence characteristics of the genus Pandoraea in lung epithelial cells
}

Correspondence
Siobhán McClean
siobhan.mcclean@ittdublin.ie

Received 31 July 2007

Accepted 21 September 2007

\author{
Emma Caraher, ${ }^{1}$ Jonathan Collins, ${ }^{2,3}$ Gillian Herbert, ${ }^{1}$ Philip G. Murphy, ${ }^{2,3}$ \\ Charles G. Gallagher, ${ }^{4}$ Mary J. Crowe, ${ }^{4}$ Máire Callaghan ${ }^{1}$ \\ and Siobhán McClean ${ }^{1}$
}

\author{
${ }^{1}$ Department of Applied Science and National Institute of Cellular Biotechnology, Institute of \\ Technology, Tallaght, Dublin 24, Ireland \\ ${ }^{2}$ Adelaide, Meath and National Children's Hospital, Tallaght, Dublin 24, Ireland \\ ${ }^{3}$ Department of Clinical Microbiology, Trinity College, Dublin, Ireland \\ ${ }^{4}$ St Vincent's University Hospital, Dublin 4, Ireland
}

\begin{abstract}
Pandoraea species are emerging opportunistic pathogens capable of causing chronic lung infections in cystic fibrosis patients. This study examined the interactions of 17 Pandoraea isolates from the five identified species (Pandoraea apista, Pandoraea norimbergensis, Pandoraea pulmonicula, Pandoraea sputorum and Pandoraea pnomenusa) plus two Pandoraea genomospecies isolates with lung epithelial cells and their ability to form biofilms in vitro. Only three isolates showed an ability to invade A549 lung epithelial cells, and only one isolate was able to form biofilms. In contrast, all isolates triggered a pronounced pro-inflammatory response, with elevation of both interleukin (IL)-6 (two- to 19-fold) and IL-8 (10- to 50-fold) above that observed for a control strain of Escherichia coli. This property is likely to be a major factor in the pathogenesis of the genus.
\end{abstract}

\section{INTRODUCTION}

Cystic fibrosis (CF) is the one of the most common genetically inherited diseases in Caucasians, resulting in lung disease characterized by thickened mucus secretions in the lung, leading to recurrent and persistent respiratory infections. A hierarchy of opportunistic pathogens colonize the CF lung throughout life, often culminating in chronic infection with Pseudomonas aeruginosa and, less frequently, Burkholderia cepacia complex (Bcc) organisms. On occasion, other opportunistic Gram-negative bacteria are encountered, including various Enterobacteriaceae, Achromobacter xylosoxidans and Stenotrophomonas maltophilia (Gibson et al., 2003). Although uncommon, members of the genus Pandoraea have been isolated from the lungs of CF patients. The genus comprises five named species, Pandoraea apista, Pandoraea norimbergensis, Pandoraea pulmonicula, Pandoraea sputorum and Pandoraea pnomenusa (Coenye et al., 2000), and at least three other, as yet unnamed, genomospecies (Daneshvar et al., 2001).

Pandoraea are motile, aerobic, non-fermentative, Gramnegative rods, but, as they are able to grow on Bcc-selective agar, they may be misidentified as Bcc organisms or Ralstonia spp., although they are taxonomically distinct

Abbreviations: Bcc, Burkholderia cepacia complex; CF, cystic fibrosis; IL, interleukin; MBC, minimum bactericidal concentration. from these (Coenye et al., 2000). Pandoraea spp. have been isolated from the lungs and from blood cultures of CF patients, supporting an invasive role for this pathogen (Atkinson et al., 2006; Daneshvar et al., 2001), and from other patients (Daneshvar et al., 2001; Stryjewski et al., 2003), and some strains may be transmissible between patients (Jorgensen et al., 2003). Colonization with $P$. apista has been associated with a decline in lung function in certain patients (Atkinson et al., 2006; Jorgensen et al., 2003) and in the formation of high antibody titres (Jorgensen et al., 2003), but the impact and clinical significance of colonization with these organisms remains ambiguous.

A number of virulence factors have been associated with clinical virulence of Ps. aeruginosa and Bcc, including stimulation of pro-inflammatory cytokine secretion, biofilm formation and, in the case of Bcc, the ability to invade lung epithelial cells, which may contribute to the persistence of the strains in the CF lung (Burns et al., 1996; Cieri et al., 2002; Duff et al., 2006). Indeed, biofilm formation by Ps. aeruginosa and Bcc has been specifically associated with increased resistance to antibiotics and maintenance of the bacteria in the lung (Caraher et al., 2007a; Chernish \& Aaron, 2003; Hoiby et al., 2001; Yu \& Head, 2002). Here, we report the results of studies on the interaction of a series of Pandoraea strains with lung epithelial cells and their ability to form biofilms in vitro, in 
order to gain insight into their virulence potential in $\mathrm{CF}$ lung infections.

\section{METHODS}

Bacterial strains and growth. The origins of the Pandoraea strains are listed in Table 1. The non-Irish isolates were purchased from BCCM/LMG. E. coli strain NCIB 9485 was used as a negative control in all experiments. All isolates were routinely grown on Luria-Bertani (LB) agar or in $\mathrm{LB}$ broth at $37^{\circ} \mathrm{C}$, and growth curves were determined by incubating bacteria in LB broth $\left(\mathrm{pH} \mathrm{7.0)}\right.$ at $37{ }^{\circ} \mathrm{C}$ with agitation at 200 r.p.m. Aliquots were removed at specific time intervals and $\mathrm{OD}_{600}$ was measured. Serial dilutions were made and aliquots $(50 \mu \mathrm{l})$ were plated on LB agar plates, which were incubated at $37{ }^{\circ} \mathrm{C}$ for $48 \mathrm{~h}$ to determine viable counts, expressed as c.f.u. $\mathrm{ml}^{-1}$.

Cell lines. A549 cells (passages 89 to 109) were purchased from the European Collection of Cell Cultures (ECACC) and maintained in RPMI 1640 supplemented with $5 \mathrm{mM}$ L-glutamine and $5 \mathrm{mM}$ HEPES with $10 \%$ fetal bovine serum, without antibiotics.

Measurement of interleukin (IL)-6 and IL-8. Cells were seeded on 24 -well plates $\left(4 \times 10^{5}\right.$ cells per well), cultured for $24 \mathrm{~h}$ at $37{ }^{\circ} \mathrm{C}$ in $5 \% \mathrm{CO}_{2}$ and incubated with test strains at an m.o.i. of 50 bacteria per cell for $24 \mathrm{~h}$. Cells were washed, the medium was replaced and plates were incubated for a further $24 \mathrm{~h}$. The supernatants were removed, filtered through a $0.45 \mu \mathrm{m}$ pore-size membrane to remove bacteria, and frozen prior to ELISA. The levels of IL- 6 and IL-8 in supernatants (diluted $1: 10$ ) were determined by ELISA (R\&D Systems) according to the manufacturer's instructions.

Table 1. Origins of the Pandoraea strains used in this study

\begin{tabular}{|c|c|c|}
\hline Species/isolate & Source & $\begin{array}{l}\text { Doubling } \\
\text { time }(h)\end{array}$ \\
\hline \multicolumn{3}{|l|}{ P. apista } \\
\hline LMG $16407^{\mathrm{T}}$ & CF lung (Denmark) & 1.23 \\
\hline LMG 16408 & CF lung (Denmark) & 0.88 \\
\hline LMG 16411 & CF lung (Denmark) & 1.44 \\
\hline LMG 16409 & CF lung (Denmark) & 1.12 \\
\hline $9963 / 95$ & CF lung (Denmark) & 1.26 \\
\hline 9967B/97 & CF lung (Denmark) & 1.15 \\
\hline \multicolumn{3}{|l|}{ P. pulmonicula } \\
\hline LMG 18107 & CF lung (USA) & 0.80 \\
\hline LMG 18108 & CF lung (USA) & 1.63 \\
\hline RL0345 & CF lung (Ireland) & 0.72 \\
\hline RL0308 & CF lung (Ireland) & 2.20 \\
\hline S8228 & CF lung (Ireland) & 1.48 \\
\hline S7633 & CF lung (Ireland) & 0.86 \\
\hline S7177 & CF lung (Ireland) & 1.02 \\
\hline \multicolumn{3}{|l|}{ P. pnomenusa } \\
\hline LMG $18087^{\mathrm{T}}$ & CF lung (UK) & 1.00 \\
\hline LMG 18817 & CF lung (USA) & 0.82 \\
\hline \multicolumn{3}{|l|}{ P. norimbergensis } \\
\hline LMG 13019 & Blood culture (Belgium) & 1.10 \\
\hline \multicolumn{3}{|l|}{ P. sputorum } \\
\hline LMG $18819^{\mathrm{T}}$ & CF lung (USA) & 1.12 \\
\hline \multicolumn{3}{|c|}{ Pandoraea genomospecies } \\
\hline S0293 & CF lung (Ireland) & 0.88 \\
\hline S8166 & CF lung (Ireland) & 1.58 \\
\hline
\end{tabular}

Antibiotic susceptibility tests. In order to establish the appropriate antibiotic concentrations required to kill extracellular bacteria in cell invasion assays, the MIC and minimum bactericidal concentration (MBC) were determined following exposure of strains to antibiotic for $2 \mathrm{~h}$. An inoculum of $10^{6}$ c.f.u. $\mathrm{ml}^{-1}$ of each strain was added to a 96-well plate containing serial dilutions of antibiotic in LB broth. The plate was incubated at $37{ }^{\circ} \mathrm{C}$ for $24 \mathrm{~h}$ and the $\mathrm{OD}_{600}$ was read to give the MIC. In addition, $50 \mu \mathrm{l}$ of the cultures that had been exposed to antibiotic for $2 \mathrm{~h}$ was spread on LB agar and incubated for $48 \mathrm{~h}$ to establish the $\mathrm{MBC}$ of the antibiotic.

Invasion of epithelial cells. Invasion studies were carried out on A549 epithelial cells using an adaptation of the method of Martin \& Mohr (2000). Cells were seeded on 24 -well plates $\left(4 \times 10^{5}\right.$ cells per well), cultured for $24 \mathrm{~h}$ at $37{ }^{\circ} \mathrm{C}$ in $5 \% \mathrm{CO}_{2}$ and incubated with bacterial suspensions at an m.o.i. of 50 for $2 \mathrm{~h}$. The cells were washed three times with PBS and treated with $1 \mathrm{mg}$ amikacin $\mathrm{ml}^{-1}$ for $2 \mathrm{~h}$ to kill any remaining extracellular bacteria. Intracellular bacteria were released by lysis with $0.5 \%$ Triton X-100/50 mM EDTA and invasion was quantified by viable counts on LB agar. The percentage invasion was determined as follows: (c.f.u. $\mathrm{ml}^{-1}$ recovered from lysed cells/ c.f.u. applied to cells) $\times 100$. Prior to lysis, the medium was removed and cultured in LB to ensure that no extracellular bacteria survived the antibiotic treatment.

Biofilm formation. Biofilm formation was examined by determining the ability of Pandoraea strains to form biofilms on microtitre plates (Caraher et al., 2007a). Briefly, LB broth (100 $\mu$ l per well) was inoculated with mid-exponential phase cultures $\left(\mathrm{OD}_{600} \sim 0.6\right)$ of individual strains at $1 \times 10^{6}$ or $1 \times 10^{7}$ c.f.u. $\mathrm{ml}^{-1}$ in quadruplicate. The plates were incubated at $37{ }^{\circ} \mathrm{C}$ for the specified times, rinsed thoroughly with water to remove non-adherent bacteria and airdried. Crystal violet $(1 \%, w / v)$ was added to each of the wells at ambient temperature for $30 \mathrm{~min}$, the plates were rinsed thoroughly and bound dye was dissolved with $95 \%$ ethanol containing $0.05 \%$ Triton X-100. The solubilized dye $(100 \mu \mathrm{l})$ was transferred to a new polystyrene microtitre plate and $A_{590}$ was determined in a Tecan plate reader. Biofilm formation was defined as those wells that had $A_{590}>0.05$.

Statistical analysis. Comparison of the invasiveness of the strains against the negative control was carried out using the Holm-Sidak method for multiple comparisons. Values of $P<0.05$ were deemed to be significant in all comparisons.

\section{RESULTS AND DISCUSSION}

\section{Pandoraea strains elicit a potent pro-inflammatory response}

Chronic lung inflammation is largely responsible for the morbidity in CF. In particular, accumulation of proinflammatory cytokines such as IL-8 and IL-6 results in lung tissue damage (Gibson et al., 2003). We examined whether Pandoraea strains were able to trigger such a response, which could contribute to their pathogenesis in the lung. All 19 Pandoraea strains examined stimulated pro-inflammatory cytokines, in particular IL-8. Secretion of IL-8 exceeded $10 \mathrm{ng} \mathrm{ml}^{-1}$ following exposure of A549 cells to all strains, with the exception of $P$. norimbergensis strain LMG 13019 and Pandoraea genomospecies S8166 (Fig. 1a). These latter strains, however, also elicited a significant host response, with secretion of IL-8 over nine 
(a)

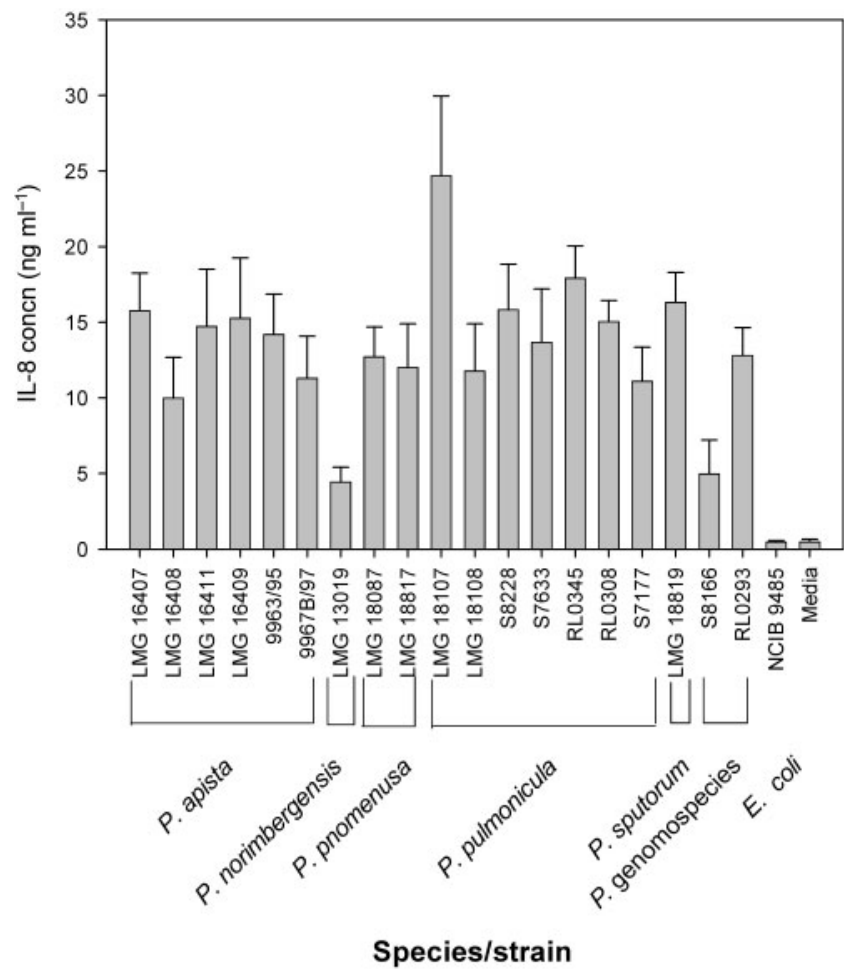

(b)

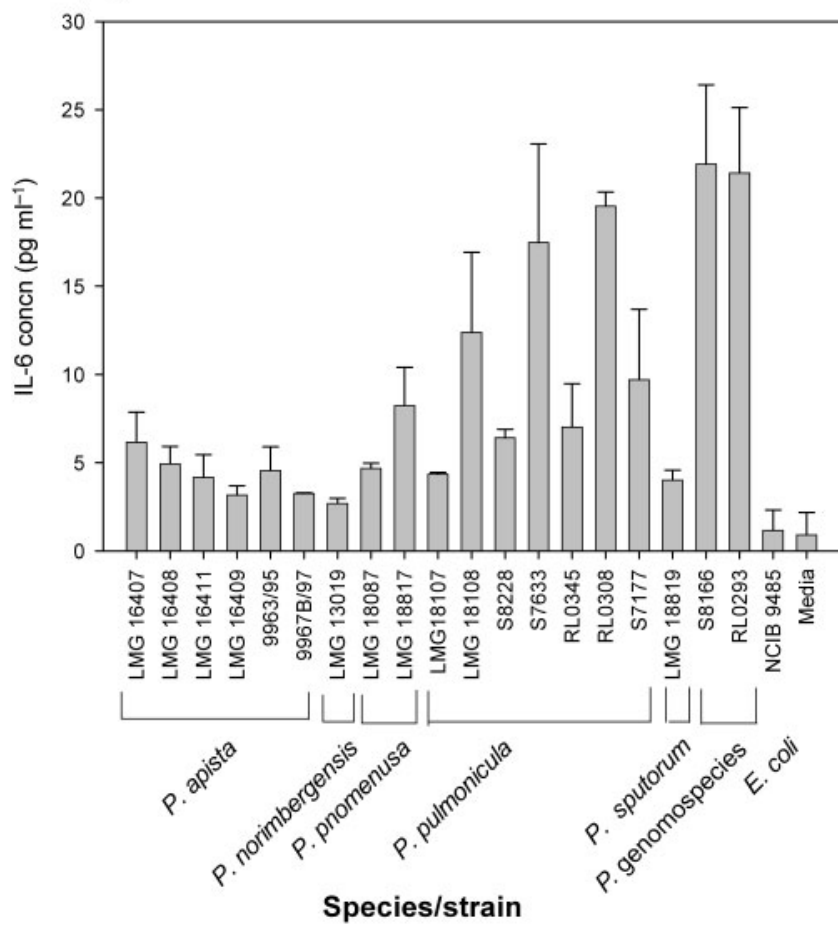

Fig. 1. Secretion of proinflammatory cytokines IL-8 (a) and II-6 (b) from lung epithelial cells following stimulation with Pandoraea strains or an E. coli strain, or with medium only. Cytokine levels were measured by ELISA and results are expressed as mean $\pm S D$ of three independent assays carried out in duplicate. times greater than that observed for E. coli. All Pandoraea strains also stimulated IL-6 secretion (Fig. 1b), although levels of secretion of this cytokine were less dramatic than those of IL-8. Maximal levels were secreted by $P$. pulmonicula strain RL0308 and by Pandoraea genomospecies isolates S8166 and RL0293 (17- to 19-fold greater than the levels secreted by $E$. coli) and were lowest in $P$. norimbergensis isolate LMG 13019 (twofold greater than $E$. coli). This finding suggests that this is a potential mechanism of pathogenesis for these organisms. Comparable increases in IL-8 and IL-6 production have been observed following colonization with the CF pathogens Ps. aeruginosa and Bcc (Bonfield et al., 1995; Palfreyman et al., 1997) and further amplify the inflammatory response in the lungs of CF patients. Indeed, the IL8 secretion elicited by Bcc isolates is of the same order as that observed for Pandoraea in this study (Palfreyman et al., 1997). However, whilst there was much more variability in IL-8 secretion among different species within the Bcc, the levels induced by the Pandoraea strains in this study were quite comparable.

IL-6 and IL-8 are constitutively upregulated in the CF airway and both are found early in nasal and bronchoalveolar lavage fluids of infants and children with CF compared with those without the defect (Khan et al., 1995; Muhlebach \& Noah, 2002; Noah et al., 1997). Upregulation of these cytokines in CF patients has been shown to lead to increased neutrophil recruitment and further enhancement of inflammation in the lung (De Rose, 2002), whilst IL-6 is also important for the augmentation of antibody production and the acute-phase response (Khair et al., 1996) The dramatic expression of these two interleukins by all Pandoraea strains examined indicates that this is an important mechanism of virulence for this genus and is likely to contribute to the pathogenesis of colonized CF patients. Many patients colonized with Pandoraea are cocolonized with other CF pathogens, in particular Ps. aeruginosa (Jorgensen et al., 2003), and the stimulation of inflammatory effects by Pandoraea would most likely aggravate the chronic inflammation that results from infection with other pathogens.

\section{Amikacin is a suitable antibiotic for invasion assays}

The integrity of the in vitro invasion assay depends on the killing of all extracellular bacteria prior to cell lysis. We previously examined invasion of Bcc strains with a combination of ceftazidime and amikacin (Caraher et al., 2007a; Duff et al., 2006), and therefore in this study we tested all strains for susceptibility to these agents $(1 \mathrm{mg}$ $\mathrm{ml}^{-1}$ each). All strains except for one P. apista strain (9967B/97) were killed by concentrations of $512 \mu \mathrm{g}$ amikacin $\mathrm{ml}^{-1}$ or less following a $2 \mathrm{~h}$ exposure, but the majority of strains survived exposure to ceftazidime (not shown). As a result, only amikacin $\left(1 \mathrm{mg} \mathrm{ml}^{-1}\right)$ was used in the invasion assays to kill extracellular Pandoraea strains. 
The amikacin-resistant $P$. apista strain was therefore excluded from the invasion experiments.

\section{Invasion of A549 cells by Pandoraea strains}

Invasion of epithelial cells can confer advantages on the pathogen, including the avoidance of host defence processes and protection from antimicrobial agents. Bcc organisms have the ability to invade lung epithelial cells (Cieri et al., 2002; Duff et al., 2006), and the levels of invasion of A549 cells were found to correlate with the virulence of strains in an in vivo mouse infection model (Cieri et al., 2002). We found that only three of the 18 Pandoraea strains were able to invade A549 lung epithelial cells (Fig. 2), i.e. gave percentage invasion values significantly different from that obtained for the negative-control E. coli strain $(P<0.05$ for RL0308, RL0345 and S8228). All three of these strains were $P$. pulmonicola. Two other strains appeared to be moderately invasive $(P$. pulmonicola S7177 and P. pnomenusa LMG 18087), but values were not significantly different from the control $(P=0.05)$. The remainder were classified as non-invasive.

The level of invasiveness of the three strains described above for A549 cells was comparable to that observed for a

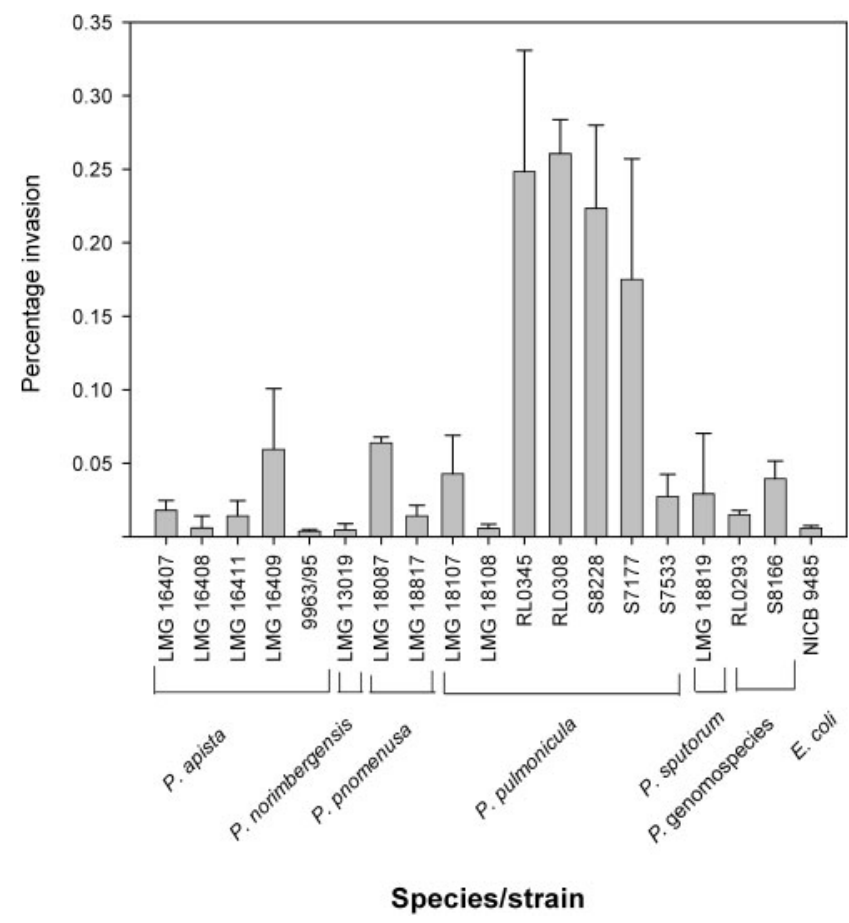

Fig. 2. Invasion of A549 lung epithelial cells by Pandoraea strains compared to that of $E$. coli strain NCIB 9485. Strains were applied to the cells for $2 \mathrm{~h}$, extracellular bacteria were killed with $1 \mathrm{mg}$ amikacin $\mathrm{ml}^{-1}$, and the c.f.u. of internalized bacteria was determined and expressed as a percentage of the bacteria applied $\left(2 \times 10^{7}\right.$ cells). Results are shown as mean percentage invasion \pm SEM for three independent assays.
Burkholderia multivorans strain (LMG 13010) and a piliated Burkholderia cenocepacia strain (C5424) (Duff et al., 2006). Overall, the ability to invade cells is not common among Pandoraea and therefore is unlikely to be a major contributor to strain virulence, in contrast to Bcc strains. The small number of invasive isolates and the fact that the patients were co-colonized with other pathogens make it difficult to correlate invasiveness with clinical outcome in patients. However, one of the invasive isolates, RL0345, was acquired from a pre-transplant patient who is doing well post-transplant. This patient was co-colonized with Ps. aeruginosa but experienced significant decline in the first 12 months following first identification with Pandoraea (forced expiratory volume in $1 \mathrm{~s}$ declined from 75 to $55 \%$, body mass dropped from 45 to $38 \mathrm{~kg}$ ). The other invasive $P$. pulmonicula isolate, RL0308, was isolated from a patient who was also co-colonized with $P$ s. aeruginosa and who subsequently died, aged 19 years (J. Collins, unpublished data). The third invasive isolate, S8228, was identified in an Irish patient who experienced no clinical deterioration following Pandoraea colonization.

\section{Pandoraea does not readily form biofilms}

Both Bcc and Ps. aeruginosa form strong biofilms, which have been associated with increased antibiotic resistance (Caraher et al., 2007b; Desai et al., 1998; Johnson et al., 2004; Moskowitz et al., 2004). We found only one Pandoraea strain, P. pulmonicula S7177, that was capable of forming biofilms (Fig. 3). This property was not related to the growth rate of the strain, as its doubling time of $61 \mathrm{~min}$ was comparable to that of other isolates that did not form biofilms (Table 1). Another P. pulmonicula strain, RL0345, formed a weak biofilm, with $A_{590}$ values just above the threshold of 0.05 at all time points examined. The OD values of all other strains were $<0.05$ at $72 \mathrm{~h}$ (not shown). These findings suggest that biofilm formation is not a major virulence factor or a likely source of antimicrobial resistance in vivo.

Despite its emergence in CF patients, little has been published on the disease burden and pathogenicity of Pandoraea spp. Chronic lung infection of six patients with a single clone of $P$. apista was associated with decreased lung function (Jorgensen et al., 2003) and two different $P$. apista strains were implicated in the deterioration of lung function in two patients, but it was unclear whether this was due directly to the presence of these organisms (Atkinson et al., 2006). P. apista was also identified in blood cultures and sputum of a 16-year-old CF patient (Johnson et al., 2004).

P. pnomenusa, however, was identified as the causative agent of sepsis and subsequent multiple organ failure in a posttransplant pulmonary sarcoidosis patient (Stryjewski et al., 2003). Furthermore, cross-infection with Pandoraea spp. has been reported in CF centres (Coenye et al., 2001; Jorgensen et al., 2003). Pandoraea has also emerged recently in the Irish CF population, with Pandoraea pulmonicula and Pandoraea 


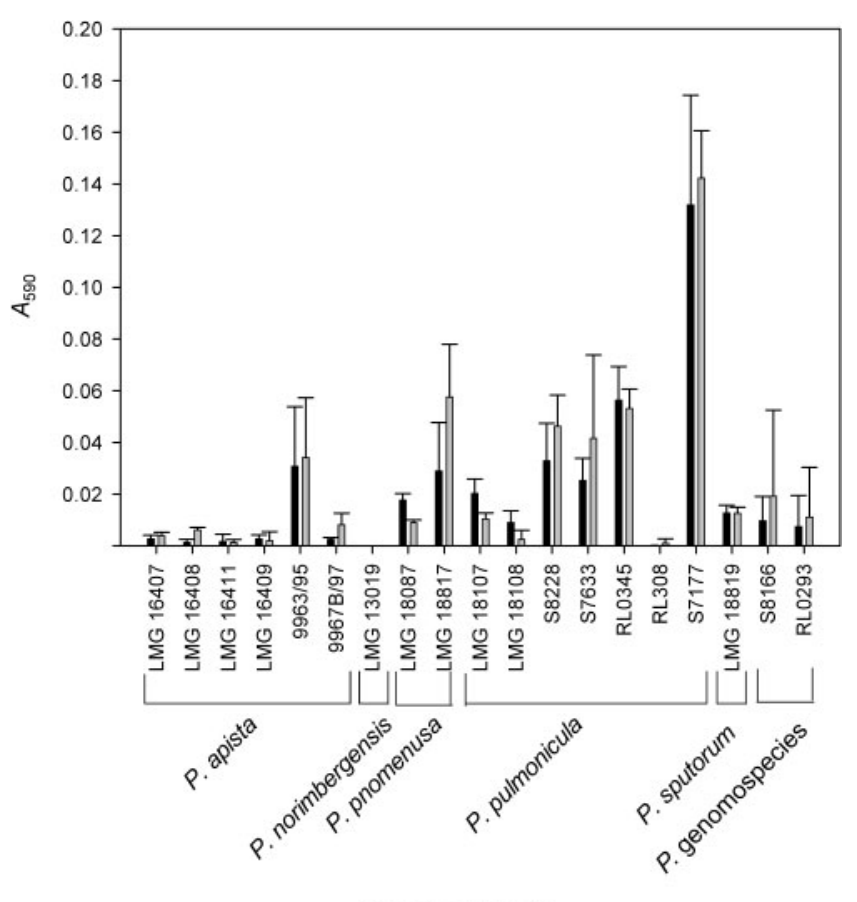

Species/strain

Fig. 3. Biofilm formation by members of the genus Pandoraea at 24 and $48 \mathrm{~h}$. Strains were inoculated at a concentration of $10^{7}$ c.f.u. $\mathrm{ml}^{-1}$ in 96 -well plates, and the biofilms were stained at $24 \mathrm{~h}$ (filled bars) and $48 \mathrm{~h}$ (shaded bars) with crystal violet. Bars represent the mean \pm SEM of three independent experiments.

genomospecies being the most predominantly identified (J. Collins and others, unpublished data). However, consistent with experience elsewhere, all Pandoraea-colonized Irish patients were co-colonized with other CF pathogens, making it difficult to draw correlations between clinical outcome and the in vitro analysis.

In conclusion, whilst a minority of Pandoraea strains examined were able to invade lung epithelial cells, the most dramatic and universal effect was the stimulated production of proinflammatory cytokines. Further studies are necessary to establish the significance of this property in lung infection in CF patients.

\section{ACKNOWLEDGEMENTS}

The authors would like to express their gratitude to Claire Schindler for her technical assistance at the beginning of this study. This work was funded by the Post-graduate R\&D Skills Programme (PRDSP) and the Programme for Research in Third Level Institutions (PRTLI) administered by the Higher Education Authority (HEA), Ireland.

\section{REFERENCES}

Atkinson, R. M., Lipuma, J. J., Rosenbluth, D. B. \& Dunne, W. M., Jr (2006). Chronic colonization with Pandoraea apista in cystic fibrosis

patients determined by repetitive-element-sequence PCR. J Clin Microbiol 44, 833-836.

Bonfield, T. L., Panuska, J. R., Konstan, M. W., Hilliard, K. A., Hilliard, J. B., Ghnaim, H. \& Berger, M. (1995). Inflammatory cytokines in cystic fibrosis lungs. Am J Respir Crit Care Med 152, 2111-2118.

Burns, J. L., Jonas, M., Chi, E. Y., Clark, D. K., Berger, A. \& Griffith, A. (1996). Invasion of respiratory epithelial cells by Burkholderia (Pseudomonas) cepacia. Infect Immun 64, 4054-4059.

Caraher, E., Duff, C., Mullen, T., Mc Keon, S., Murphy, P., Callaghan, M. \& McClean, S. (2007a). Invasion and biofilm formation of Burkholderia dolosa is comparable with Burkholderia cenocepacia and Burkholderia multivorans. J Cyst Fibros 6, 49-56.

Caraher, E., Reynolds, G., Murphy, P., McClean, S. \& Callaghan, M. (2007b). Comparison of antibiotic susceptibility of Burkholderia cepacia complex organisms when grown planktonically or as biofilm in vitro. Eur J Clin Microbiol Infect Dis 26, 213-216.

Chernish, R. N. \& Aaron, S. D. (2003). Approach to resistant Gramnegative bacterial pulmonary infections in patients with cystic fibrosis. Curr Opin Pulm Med 9, 509-515.

Cieri, M. V., Mayer-Hamblett, N., Griffith, A. \& Burns, J. L. (2002). Correlation between an in vitro invasion assay and a murine model of Burkholderia cepacia lung infection. Infect Immun 70, 1081-1086.

Coenye, T., Falsen, E., Hoste, B., Ohlen, M., Goris, J., Govan, J. R., Gillis, M. \& Vandamme, P. (2000). Description of Pandoraea gen. nov. with Pandoraea apista sp. nov., Pandoraea pulmonicola sp. nov., Pandoraea pnomenusa sp. nov., Pandoraea sputorum sp. nov. and Pandoraea norimbergensis comb. nov. Int J Syst Evol Microbiol 50, 887-899.

Coenye, T., Liu, L., Vandamme, P. \& LiPuma, J. J. (2001). Identification of Pandoraea species by $16 \mathrm{~S}$ ribosomal DNA-based PCR assays. J Clin Microbiol 39, 4452-4455.

Daneshvar, M. I., Hollis, D. G., Steigerwalt, A. G., Whitney, A. M., Spangler, L., Douglas, M. P., Jordan, J. G., MacGregor, J. P., Hill, B. C. \& other authors (2001). Assignment of CDC weak oxidizer group 2 (WO-2) to the genus Pandoraea and characterization of three new Pandoraea genomospecies. J Clin Microbiol 39, 1819-1826.

De Rose, V. (2002). Mechanisms and markers of airway inflammation in cystic fibrosis. Eur Respir J 19, 333-340.

Desai, M., Buhler, T., Weller, P. H. \& Brown, M. R. (1998). Increasing resistance of planktonic and biofilm cultures of Burkholderia cepacia to ciprofloxacin and ceftazidime during exponential growth. J Antimicrob Chemother 42, 153-160.

Duff, C., Murphy, P. G., Callaghan, M. \& McClean, S. (2006). Differences in invasion and translocation of Burkholderia cepacia complex species in polarised lung epithelial cells in vitro. Microb Pathog 41, 183-192.

Gibson, R. L., Burns, J. L. \& Ramsey, B. W. (2003). Pathophysiology and management of pulmonary infections in cystic fibrosis. Am J Respir Crit Care Med 168, 918-951.

Hoiby, N., Krogh Johansen, H., Moser, C., Song, Z., Ciofu, O. \& Kharazmi, A. (2001). Pseudomonas aeruginosa and the in vitro and in vivo biofilm mode of growth. Microbes Infect 3, 23-35.

Johnson, L. N., Han, J. Y., Moskowitz, S. M., Burns, J. L., Qin, X. \& Englund, J. A. (2004). Pandoraea bacteremia in a cystic fibrosis patient with associated systemic illness. Pediatr Infect Dis J 23, 881-882.

Jorgensen, I. M., Johansen, H. K., Frederiksen, B., Pressler, T., Hansen, A., Vandamme, P., Hoiby, N. \& Koch, C. (2003). Epidemic spread of Pandoraea apista, a new pathogen causing severe lung disease in cystic fibrosis patients. Pediatr Pulmonol 36, 439-446.

Khair, O. A., Davies, R. J. \& Devalia, J. L. (1996). Bacterial-induced release of inflammatory mediators by bronchial epithelial cells. Eur Respir J 9, 1913-1922. 
Khan, T. Z., Wagener, J. S., Bost, T., Martinez, J., Accurso, F. J. \& Riches, D. W. (1995). Early pulmonary inflammation in infants with cystic fibrosis. Am J Respir Crit Care Med 151, 1075-1082.

Martin, D. W. \& Mohr, C. D. (2000). Invasion and intracellular survival of Burkholderia cepacia. Infect Immun 68, 24-29.

Moskowitz, S. M., Foster, J. M., Emerson, J. \& Burns, J. L. (2004). Clinically feasible biofilm susceptibility assay for isolates of Pseudomonas aeruginosa from patients with cystic fibrosis. J Clin Microbiol 42, 1915-1922.

Muhlebach, M. S. \& Noah, T. L. (2002). Endotoxin activity and inflammatory markers in the airways of young patients with cystic fibrosis. Am J Respir Crit Care Med 165, 911-915.
Noah, T. L., Black, H. R., Cheng, P. W., Wood, R. E. \& Leigh, M. W. (1997). Nasal and bronchoalveolar lavage fluid cytokines in early cystic fibrosis. J Infect Dis 175, 638-647.

Palfreyman, R. W., Watson, M. L., Eden, C. \& Smith, A. W. (1997). Induction of biologically active interleukin-8 from lung epithelial cells by Burkholderia (Pseudomonas) cepacia products. Infect Immun 65, 617-622.

Stryjewski, M. E., LiPuma, J. J., Messier, R. H., Jr, Reller, L. B. \& Alexander, B. D. (2003). Sepsis, multiple organ failure and death due to Pandoraea pnomenusa infection after lung transplantation. J Clin Microbiol 41, 2255-2257.

Yu, H. \& Head, N. E. (2002). Persistent infections and immunity in cystic fibrosis. Front Biosci 7, d442-457. 